\title{
Statistical Analysis of College Students' Common Diseases in Tianjin Agricultural University from the Perspective of Male and Female
}

\author{
Hongjun Teng ${ }^{1, a^{*}}$, Zhenbo Bao ${ }^{2, b}$ and Fan Yang ${ }^{2, c}$ \\ ${ }^{1}$ Clinic, Tianjin Agricultural University, Tianjin, China, 300384 \\ ${ }^{2}$ Engineering and Technology College, Tianjin Agricultural University, Tianjin, China, 300384 \\ a*hongjun-teng@163.com, bbaozhenbo@sohu.com, 'fanyang20062800@126.com
}

\section{Keywords: College students; Common diseases; Statistical Analysis; Male and Female}

\begin{abstract}
To continuously improve the college students' physical health is an important task of higher education. From September 2016 to January 2018, statistics were collected on common diseases of male and female college students who went to Tianjin Agricultural University School Hospital. Statistics found: The 10 common diseases of male college students with a high proportion of distribution are cold, upper respiratory tract infections (pharyngitis, tonsils, etc.), sprain, fire, gastroenteritis, bronchitis, skin allergy, toothache, stomachache and eye fatigue. The 12 common diseases of female college students with a high proportion of distribution are cold, upper respiratory tract infections (pharyngitis, tonsils, etc.), sprain, fire, skin allergy, conjunctivitis, bronchitis, toothache, otitis media, stomachache, gastroenteritis and eye fatigue. The overall number of female college students with common diseases (975 cases) is higher than the total number of common diseases of male college students (670 cases). The number of male college students with gastroenteritis (40 cases) is higher than that of female university students ( 21 cases). The number of female college students suffering from conjunctivitis (51 cases) is significantly higher than that of male college students (11 cases). The number of female college students with common diseases of otitis media (24 cases) is relatively high, and male college students have almost no such disease. Through the statistical analysis of male and female college students' common diseases, it has a positive effect on guiding health education.
\end{abstract}

\section{Introduction}

Promoting the healthy growth of college students is the starting point and the foothold of all the work of the school. It is an important task of higher education to constantly improve the health level of college students. I have been engaged in the diagnosis and treatment, health care and health education in the comprehensive clinic for more than 10 years in the university hospital. I realize that through the summary of the common disease regularity of the college students and the targeted health education, it has a positive effect on promoting the knowledge of disease prevention and health care and reducing the occurrence of college students' common diseases [1,2]. From September 2016 to January 2018, the common diseases of college students who went to Tianjin Agricultural University School Hospital were analyzed from the perspective of male and female. The distribution rules of male and female college students' common diseases were mastered to provide a reference for better health care and health education $[3,4]$.

\section{Statistical Analysis of Male College Students' Common Diseases in Tianjin Agricultural University}

From September 2016 to January 2018, statistics were conducted on male college students' common diseases treated by out-patients at Tianjin Agricultural University School Hospital, and the distribution of male college students' common diseases in Tianjin Agricultural University was summarized. There were 670 male college students who came to the clinic. The rate of return visits was not within the statistical range. Male college students were between 18 and 25 years old. Common diseases of male college students are: cold, upper respiratory tract infections (pharyngitis, tonsils, etc.), sprains, fire, 
gastroenteritis, bronchitis, skin allergies, toothache, stomachache, eye fatigue, conjunctivitis, headache, dizziness, hypertension and hypotension, etc. 13 common diseases. The names, distribution and proportion of common diseases are shown in Table $1[5,6]$.

Table 1 The distribution and proportion of male college students' common diseases in Tianjin Agricultural University

\begin{tabular}{|c|c|c|c|}
\hline No. & Common diseases & Cases & Proportion[\%] \\
\hline 1 & Cold & 205 & 30.6 \\
\hline 2 & Upper respiratory tract infections (pharyngitis, tonsils, etc.) & 145 & 21.64 \\
\hline 3 & Sprain & 80 & 11.94 \\
\hline 4 & Fire & 59 & 8.81 \\
\hline 5 & Gastroenteritis & 40 & 5.97 \\
\hline 6 & Bronchitis & 37 & 5.52 \\
\hline 7 & Skin allergy & 33 & 4.93 \\
\hline 8 & Toothache & 19 & 2.84 \\
\hline 9 & Stomachache & 14 & 2.09 \\
\hline 10 & Eye fatigue & 13 & 1.94 \\
\hline 11 & Conjunctivitis & 11 & 1.64 \\
\hline 12 & Headache, dizziness, hypertension and hypotension & 8 & 1.19 \\
\hline 13 & Beriberi & 6 & 0.9 \\
\hline Total & & 670 & 100 \\
\hline
\end{tabular}

The 10 common diseases of male college students with a high proportion of distribution are cold, upper respiratory tract infections (pharyngitis, tonsils, etc.), sprain, fire, gastroenteritis, bronchitis, skin allergy, toothache, stomachache and eye fatigue, and their distribution proportions are shown in Fig 1.

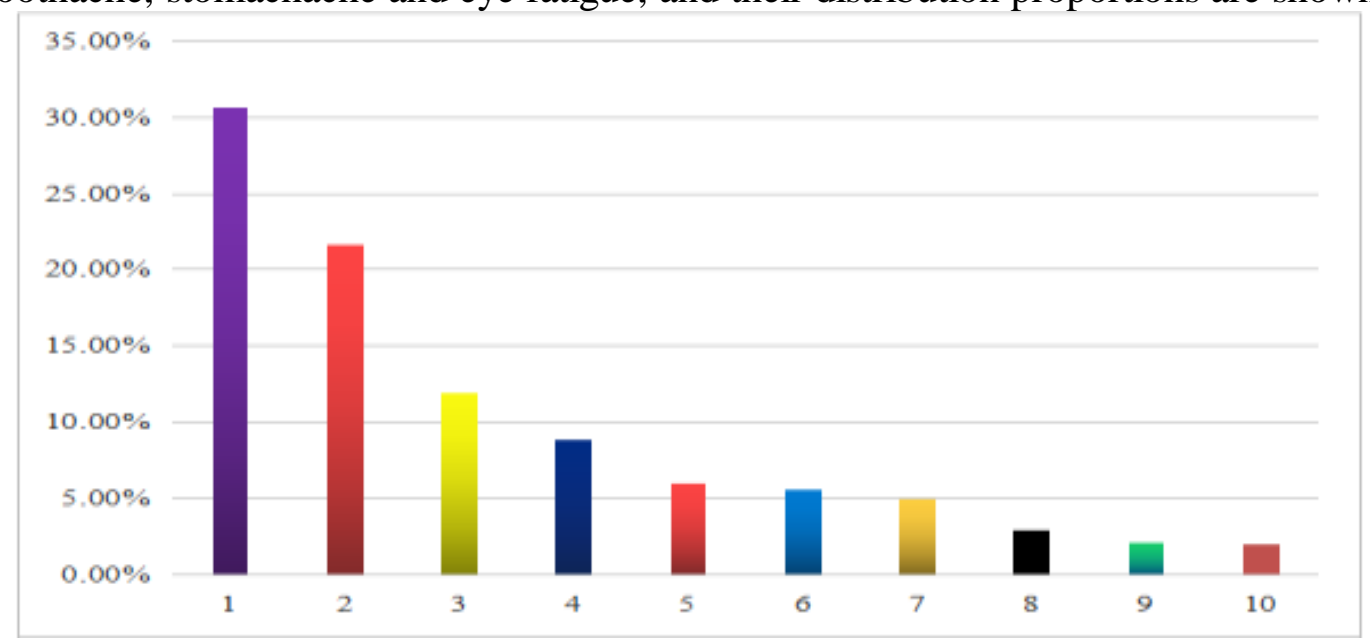

1.Cold 2.Upper respiratory tract infections (pharyngitis, tonsils, etc.) 3.Sprain 4.Fire 5.Gastroenteritis 6.Bronchitis 7.Skin allergy 8.Toothache 9.Stomachache 10.Eye fatigue

Figure 1. The distribution and proportion of male students' common diseases in Tianjin Agricultural University

\section{Statistical Analysis of Female College Students' Common Diseases in Tianjin Agricultural University}

From September 2016 to January 2018, statistics were conducted on the common diseases of female college students treated by out-patients at Tianjin Agricultural University School Hospital, and the distribution of female college students' common diseases in Tianjin Agricultural University was summarized. From September 2016 to January 2018, there were 975 female college students who came to the clinic. The rate of return visits was not within the statistical range. Female college students were 
between 18 and 25 years old. Common diseases of female college students are: cold, upper respiratory tract infections (pharyngitis, tonsils, etc.), sprains, fire, skin allergies, conjunctivitis, bronchitis, toothache, otitis media, stomachache, gastroenteritis, eye fatigue, etc. 15 common diseases. The names, distribution and proportion of common diseases are shown in Table $2[7,8]$.

Table 2 The distribution and proportion of female students' common diseases in Tianjin Agricultural University

\begin{tabular}{|c|c|c|c|}
\hline No. & Common diseases & Cases & Proportion[\%] \\
\hline 1 & Cold & 270 & 27.69 \\
\hline 2 & Upper respiratory tract infections (pharyngitis, tonsils, etc.) & 159 & 16.31 \\
\hline 3 & Sprain & 119 & 12.21 \\
\hline 4 & Fire & 104 & 10.67 \\
\hline 5 & Skin allergy & 68 & 6.73 \\
\hline 6 & Conjunctivitis & 51 & 5.23 \\
\hline 7 & Bronchitis & 50 & 5.13 \\
\hline 8 & Toothache & 46 & 4.72 \\
\hline 9 & Otitis media & 24 & 2.46 \\
\hline 10 & Stomachache & 23 & 2.36 \\
\hline 11 & Gastroenteritis & 21 & 2.15 \\
\hline 12 & Eye fatigue & 20 & 2.05 \\
\hline 13 & Headache, dizziness, hypertension and hypotension & 9 & 0.92 \\
\hline 14 & Dysmenorrhea & 6 & 0.62 \\
\hline 15 & Beriberi & 5 & 0.51 \\
\hline Total & & & 100 \\
\hline
\end{tabular}

The 12 common diseases of female college students with a high proportion of distribution are cold, upper respiratory tract infections (pharyngitis, tonsils, etc.), sprain, fire, skin allergy, conjunctivitis, bronchitis, toothache, otitis media, stomachache, gastroenteritis and eye fatigue, and their distribution proportions are shown in Fig 2.

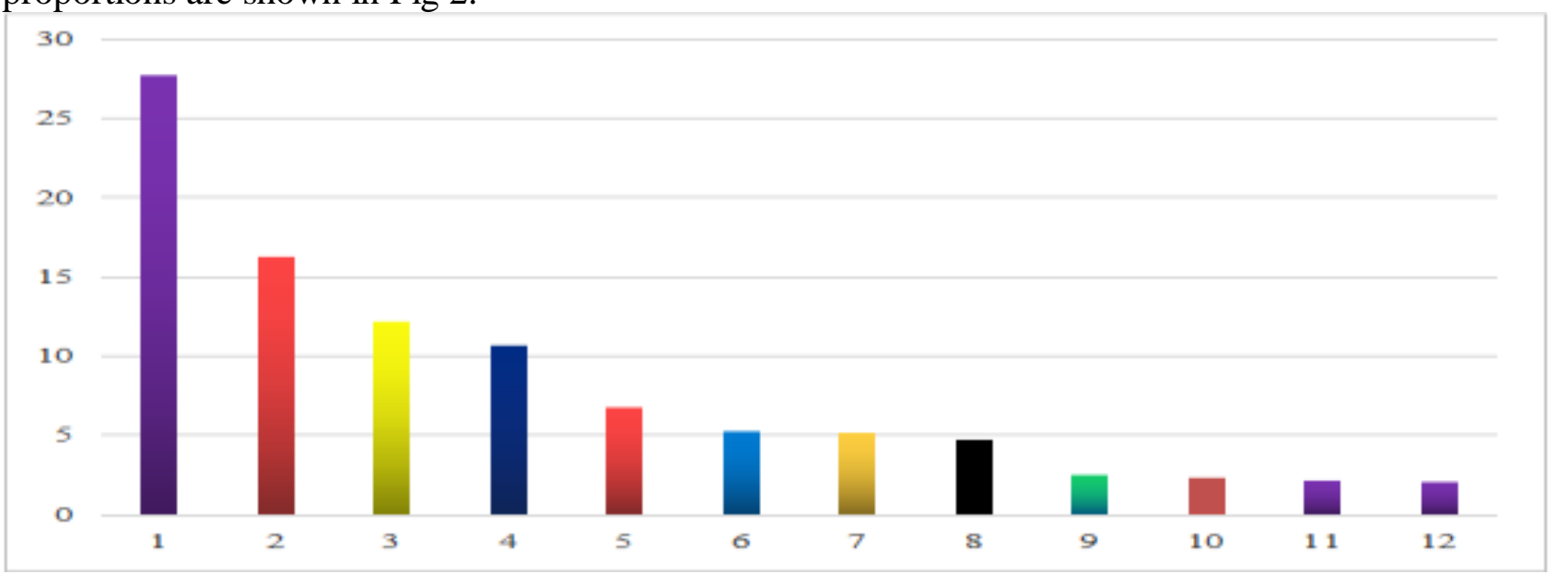

1.Cold 2.Upper respiratory tract infections (pharyngitis, tonsils, etc.) 3.Sprain

4.Fire 5.Skin allergy 6.Conjunctivitis 7.Bronchitis 8.Toothache 9.Otitis media 10.Stomachache 11.Gastroenteritis 12.Eye fatigue

Figure 2. The distribution and proportion of female students' common diseases in Tianjin Agricultural University 


\section{Statistical Analysis and Comparison of Male and Female College Students' Common Diseases in Tianjin Agricultural University}

From September 2016 to January 2018, statistics were collected on common diseases of male and female university students attending the Tianjin Agricultural University School Hospital, and the distribution patterns of college students' common diseases were summarized. The statistical results are shown in Table 3.

Table 3 The distribution and proportion of male and female students' common diseases in Tianjin Agricultural University

\begin{tabular}{|c|c|c|c|c|c|}
\hline \multirow{2}{*}{ No. } & \multirow{2}{*}{ Common diseases } & \multicolumn{2}{|c|}{ Male college students } & \multicolumn{2}{c|}{ Female college students } \\
\cline { 3 - 6 } & Cases & Proportion[\%] & Cases & Proportion[\%] \\
\hline 1 & Cold & 205 & 12.46 & 270 & 16.41 \\
\hline 2 & $\begin{array}{c}\text { Upper respiratory tract infections } \\
\text { (pharyngitis, tonsils, etc.) }\end{array}$ & 145 & 8.81 & 159 & 9.67 \\
\hline 3 & Sprain & 80 & 4.86 & 119 & 7.23 \\
\hline 4 & Fire & 59 & 3.59 & 104 & 6.32 \\
\hline 5 & Gastroenteritis & 40 & 2.43 & 21 & 1.28 \\
\hline 6 & Bronchitis & 37 & 2.25 & 50 & 3.04 \\
\hline 7 & Skin allergy & 33 & 2.01 & 68 & 4.13 \\
\hline 8 & Toothache & 19 & 1.16 & 46 & 2.80 \\
\hline 9 & Stomachache & 13 & 0.85 & 23 & 1.40 \\
\hline 10 & Eye fatigue & 11 & 0.67 & 20 & 1.22 \\
\hline 11 & Conjunctivitis & 8 & 0.49 & 51 & 3.10 \\
\hline 12 & Headache dizziness, hypertension & 6 & 9 & 0.55 \\
\hline 13 & and hypotension & 0 & 0 & 5 & 0.30 \\
\hline 14 & Beriberi & 0 & 0 & 6 & 0.37 \\
\hline 15 & Otitis media & 670 & 40.73 & 975 & 59.27 \\
\hline Total & Dysmenorrhea & & & \\
\hline
\end{tabular}

In general, the overall number of female college students with common diseases ( 975 cases) is higher than the total number of common diseases of male college students (670 cases). The number of common patients among male and female college students was: cold, upper respiratory tract infections (pharyngitis, tonsils, etc.), sprains, fire, gastroenteritis, bronchitis, skin allergies, toothache, stomachache and eye fatigue, and the number of female college students was significantly higher than that of male college students. The number of male college students with common gastroenteritis (40 cases) is higher than that of female university students (21 cases). The number of female college students suffering from conjunctivitis (51 cases) was significantly higher than that of male college students (11 cases). The number of female college students with common diseases of otitis media (24 cases) is relatively high, and male college students have almost no such disease.

\section{Summary}

Through the statistical analysis of the male and female college students' common diseases in Tianjin Agricultural University, the common diseases of 10 kinds of male college students with high distribution ratio are cold, upper respiratory tract infections (pharyngitis, tonsils, etc.), sprain, fire, gastroenteritis, bronchitis, skin allergy, toothache, stomachache and eye fatigue, and the common diseases of 12 kinds of female college students with high distribution ratio are cold, upper respiratory tract infections (pharyngitis, tonsils, etc.), sprains, fire, skin allergies, conjunctivitis, bronchitis, toothache, otitis media, stomachache, gastroenteritis and eye fatigue. The total number of female college students' common diseases is higher than that of male college students, which indicates that the overall physical fitness and 
health of male college students is higher than that of female college students. The number of male college students with gastroenteritis is higher than that of female college students, which indicates that male college students need to pay more attention to dietary health and hygiene. The number of female college students suffering from conjunctivitis and otitis media is significantly higher than the number of male college students, which is related to the excessive use of cosmetics and long hair bathing by female college students.

\section{Acknowledgements}

This work was financially supported by the Project of Tianjin Agricultural University Education and Teaching Reform Research (2016-A-04).

\section{References}

[1] W.Y. Yan, X.M. Jia: For all Health, Vol. 10 (2016) No.15, p.8.

[2] Q.B. Wang, X. Shi, Y.Y. Wei: China Medical Herald, Vol. 6 (2009) No.26, p.120.

[3] X.C. Sun: China Medical Engineering, Vol. 19 (2011) No.12, p.149-150.

[4] W.J. Liu: Journal of Guangdong University of Petrochemical Technology, Vol. 23 (2013) No.5, p.35-37.

[5] Z,H, Lu: Journal of Qiqihar University of Medical, Vol. 36 (2015) No.11, p.1664-1665.

[6] Y.H. Feng, M.Y. Wu, H.L. Chen: Journal Traditional Medicine Management, Vol. 24 (2016) No.10, p.167-169.

[7] S.M. Sun: Chin J Sch Health, Vol. 35 (2014) No.11, p.1739-1741.

[8] Q.T. Wu, J.W. Gan: Health Medicine Research and Practice, Vol. 12 (2015) No.5, p.86-88. 